${ }^{14}$ W. E. Blumberg, Phys. Rev. $119,79(1960)$.

${ }^{15}$ G. R. Khutsishvili, Usp. Fiz. Nauk 96,441 (1968)

[Sov. Phys. Usp. 11, 802 (1969)].

${ }^{16}$ J. I. Kaplan, Phys. Rev. B 3, 604 (1971).

${ }^{17}$ The theory of energy transfer has been developed by D. L. Dexter [J. Chem. Phys. 21, 836 (1953)] and D. L. Dexter and J. H. . chulman [ibid. 22, 1063 (1954)].

${ }^{18}$ From the numerical integration of Eq. (2) Kaplan (Ref. 16) finds

$$
\tau_{D}^{-1}=\frac{4\left(\frac{4}{3} \pi N C^{1 / 2}\right)\left(D / C^{1 / 3}\right)^{3 / 4}}{\left[\left(3 / 4 \pi N C^{1 / 2}\right)^{1 / 3}\left(D / C^{1 / 3}\right)^{1 / 4}-1\right]^{1 / 3}}
$$

which yields a slightly different functional dependence of $\tau_{D}^{-1}$ even though the values for $D$ are not too different from Eq. (5).

${ }^{19}$ The Pade approximant in the brackets is quite closely approximated by $\left(1+\pi^{1 / 2} z\right)^{3 / 4}$.

${ }^{20}$ R. E. McDonald and J. W. Beck, J. Appl. Phys. $\underline{40}$, 1429 (1969).

${ }^{21}$ D. S. McClure, J. Chem. Phys. 36, 2757 (1962).

${ }^{22} \mathrm{~J}$. P. van der Ziel, F. W. Ostermayer, Jr., and L. G. Van Uitert, Phys. Rev. B 2 , 4432 (1970).

${ }^{23}$ This is also the $\mathrm{Eu}^{3+}$ lifetime in the phosphate glass obtained by M. Weber, E. J. Sharpe, and J. E. Miller [J. Phys. Chem. Solids $\underline{32}, 2775$ (1971)].

\title{
Surface Mean-Square Amplitudes of Vibration for $\mathrm{NaCl}^{\dagger}$
}

\author{
T. S. Chen, G. P. Alldredge, and F. W. de Wette \\ Department of Physics, University of Texas, Austin, Texas 78712 \\ and \\ R. E. Allen \\ Department of Physics, Texas A \& $M$ University, College Station, Texas 77843 \\ (Received 15 February 1972)
}

\begin{abstract}
The mean-square amplitudes and mean-square velocities of vibration have been calculated for the (100) surface of $\mathrm{NaCl}$ with the Kellermann rigid-ion model. The calculated value of $\left\langle u_{z}^{2}\right\rangle_{\text {surf ace }} /\left\langle u_{z}^{2}\right\rangle_{\text {bulk }}$ (the $z$ direction being perpendicular to the surface) is only about 1.6 at high temperatures, as compared with calculated and experimental values ranging upward from about 2.0 for monatomic metals and noble-gas solids.
\end{abstract}

There have been a number of calculations of the mean-square amplitudes of vibration near the surfaces of monatomic crystals, ${ }^{1}$ but to our knowledge no such calculation has been performed for any ionic crystal. The mean-square amplitudes are of interest because they determine the Debye-Waller factors of the atoms or ions, and thus the temperature dependence of scattering intensities-e.g., the intensities of the "Bragg peaks" in low-energy electron diffraction (LEED). In the present paper we report calculations of the mean-square amplitudes and mean-square velocities for the (100) surface of $\mathrm{NaCl}$.

We will distinguish the $\mathrm{Na}^{+}$and $\mathrm{Cl}^{-}$ions by a label $\kappa$ and use a label $l_{3}$ to distinguish the planes of ions parallel to the surface. The mean-square amplitudes of vibration $\left\langle u_{\alpha}^{2}\left(l_{3} \kappa\right)\right\rangle$ and the meansquare velocities $\left\langle v_{\alpha}^{2}\left(l_{3} \kappa\right)\right\rangle$, with $\alpha=x, y$, or $z$, are then given in the quasiharmonic approximation by the standard expressions

$$
\begin{aligned}
\left\langle u_{\alpha}^{2}\left(l_{3} \kappa\right)\right\rangle=\frac{\hbar}{2 \overline{\mathrm{N}} M_{\kappa}} \sum_{\bar{q}, p}^{\prime}\left|\xi_{\alpha}\left(l_{3} \kappa ; \overline{\mathrm{q}} p\right)\right|^{2} & \\
& \times \frac{\operatorname{coth}\left[\hbar \omega_{p}(\overline{\mathrm{q}}) / 2 k_{B} T\right]}{\omega_{p}(\overline{\mathrm{q}})},
\end{aligned}
$$

$$
\begin{aligned}
\left\langle v_{\alpha}^{2}\left(l_{3} \kappa\right)\right\rangle=\frac{\hbar}{2 \overline{\mathrm{N}} M_{\kappa}} \sum_{\overline{\mathrm{q}}, p}^{\prime}\left|\xi_{\alpha}\left(l_{3} \kappa ; \overline{\mathrm{q}} p\right)\right|^{2} \omega_{p}(\overline{\mathrm{q}}) & \\
& \times \operatorname{coth}\left(\frac{\hbar \omega_{p}(\overline{\mathrm{q}})}{2 k_{B} T}\right) .
\end{aligned}
$$

$M_{\kappa}$ is the mass of an ion labeled by $\kappa, \overline{\mathrm{N}}$ is the number of values of the two-dimensional wave vector $\overline{\mathrm{q}}$ in the summation, $p$ is a label which distinguishes the different vibrational modes for a given value of $\bar{q}, T$ is the temperature, and $k_{B}$ is the Boltzmann constant. The vibrational frequencies $\omega_{p}(\bar{q})$ and eigenvectors $\xi_{\alpha}\left(l_{3} \kappa ; \bar{q} p\right)$ are determined by the standard eigenvalue equation

$\sum_{l_{3}^{\prime} \kappa^{\prime} \beta} D_{\alpha \beta}\left(l_{3} \kappa ; l_{3}^{\prime} \kappa^{\prime} ; \overline{\mathrm{q}}\right) \xi_{\beta}\left(l_{3}^{\prime} \kappa^{\prime} ; \overline{\mathrm{q}} p\right)=\omega_{p}^{2}(\overline{\mathrm{q}}) \xi_{\alpha}\left(l_{3} \kappa ; \overline{\mathrm{q}} p\right)$.

It is straightforward to calculate $\left\langle u_{\alpha}^{2}\left(l_{3} \kappa\right)\right\rangle$ and $\left\langle v_{\alpha}^{2}\left(l_{3} \kappa\right)\right\rangle$ by numerically solving Eqs. $(1)-(3)$ once the dynamical matrix $D_{\alpha \beta}\left(l_{3} \kappa ; l_{3}^{\prime} \kappa^{\prime} ; \overline{\mathrm{q}}\right)$ has been determined. We have calculated the dynamical matrix in exactly the same way as Tong and Maradudin, ${ }^{2}$ except for two differences described in the next paragraph. Although we are really interested in a semi-infinite crystal, we perform the calculations for a 15-layer slab in order to simplify the 
problem. The results for a slab of this thickness will closely approximate those for a semi-infinite crystal, since the bulk "bands" are rather densely populated and the surface modes are significantly affected by the finite thickness only for very small values of $\bar{q}$. We also assume the Kellermann rigid-ion (KRI) model $^{3}$ with the values of the potential parameters used by Tong and Maradudin ${ }^{2}$ (which are nominally room-temperature values). In this model, the ions are assumed to interact through a pair potential which consists of a pointcharge Coulomb potential and a nearest-neighbor Born-Mayer potential; the polarizabilities of the ions are neglected. The surface-mode spectra for the KRI model and for a shell model, which is presumably much more accurate, have been found to differ significantly. ${ }^{4}$ However, the mean-square amplitudes and mean-square velocities represent averages over all the vibrational modes, according to Eqs. (1) and (2), and should therefore not be very sensitive to details in the spectrum of vibrational modes. Furthermore, as can be seen from Eq. (1), the dominant contribution to the meansquare amplitudes comes from the acoustical modes (because of their lower frequencies) which are much better described by the KRI model than are the optical modes. We therefore feel that the KRI model should yield reasonably good estimates for these quantities (or for the surface thermodynamic functions ${ }^{5}$ ). (But see note added in proof.)

The first difference between our evaluation of the dynamical matrix and that of Ref. 2 involves the method for calculating the Coulomb lattice sums. This matter is discussed in detail elsewhere. ${ }^{6}$ In brief, we find that the method of de Wette and Schacher, ${ }^{7}$ which involves use of the incomplete gamma function, provides much better convergence than the method of Ref. 2, which involves the modified Bessel function. Consequently, we have used the "plane-wise summation method" of Ref. 7, as did Lucas. ${ }^{8}$ The second difference between Ref. 2 and the present work involves the static relaxation of the ions near the surface. Tong and Maradudin permitted both interplanar and intraplanar displacements, as is appropriate if one is interested in doing the calculations consistently for a 15-layer slab. We have, however, permitted only interplanar displacements and held the spacing within the planes fixed at the bulk value, since this is the more appropriate procedure if one is interested in approximating the results for a semi-infinite crystal as accurately as possible. The relaxation of Tong and Maradudin is positive and has a nonzero value near the center of the slab, whereas our relaxation is principally negative and tends to zero with increasing distance from the surface. However, the relaxation is small-less than $1 \%$ in either case-and not of great importance. Perhaps a more serious approximation is the assumption (here and in Ref. 2) that the $\mathrm{Na}^{+}$and $\mathrm{Cl}^{-}$ions in a given plane have equal static displacements, whereas they will in reality undergo different displacements. In the context of the KRI model, however, it is not clear that taking account of this effect would improve the accuracy of the calculation, ${ }^{9}$ and to do so would make the calculation considerably more difficult.

In using Eqs. (1) and (2), we are assuming that the quasiharmonic approximation is valid for the range of temperatures under consideration. Cowley ${ }^{10}$ has performed a calculation of the quasiharmonic and lowest-order anharmonic contributions to bulk thermodynamic quantities for $\mathrm{NaCl}$. $\mathrm{He}$ finds that, "The quasiharmonic calculation gives good agreement with experiment up to one-quarter of the melting temperature" of $1074^{\circ} \mathrm{K}$. Previous calculations of the surface mean-square amplitudes for noble-gas solids at one-half the melting temperature ${ }^{11}$ indicated that there are significant, but not enormous, anharmonic contributions at this temperature. It therefore appears that the quasiharmonic approximation should be adequate up to room temperature for the surface mean-square amplitudes in $\mathrm{NaCl}$. In the present work, we also neglect thermal expansion, which should have the effect of increasing the vibrational frequencies by about 3 or $4 \%$ between room temperature and $0^{\circ} \mathrm{K},{ }^{5}$ and thus decreasing the mean-square amplitudes at low temperature by roughly $5 \% .^{12}$

Our results for the mean-square amplitudes are shown in Figs. 1-3. ${ }^{13}$ It is interesting that the ratio $\left\langle u_{z}^{2}\right\rangle_{\text {surface }} /\left\langle u_{z}^{2}\right\rangle_{\text {bu1k }}$ reaches a value of only about 1.6 even at room temperature and above. (Here, as always, we take the $z$ direction to be normal to the surface. ) This value is considerably smaller than the values at high temperatures which have been calculated for model monatomic crystals ${ }^{1}$ and measured for monatomic metals ${ }^{1}$ and for Xe films, ${ }^{14}$ which have ranged upward from about 2.0. The relatively small value of $\left\langle u_{z}^{2}\right\rangle_{\text {surface }}$ for $\mathrm{NaCl}$ appears to be caused by the relatively high frequencies of the acoustical surface modes ${ }^{15}$ : According to Eq. (1), large values of $\omega$ for the surface modes should produce small values for the surface meansquare amplitudes.

In Fig. 4, the results for the mean-square velocities are shown. Because the equipartition theorem implies that

$$
\frac{1}{2} M_{\kappa}\left\langle v_{\alpha}^{2}\left(l_{3} \kappa\right)\right\rangle=\frac{1}{2} k_{B} T \text { for } T \rightarrow \infty,
$$

and for all $\kappa, \alpha$, and $l_{3}$, it follows that there is only a very small difference between the surface and bulk mean-square velocities at room temperature. In the present case, we have

$\left\langle v_{\alpha}^{2}\left(l_{3} \mathrm{Na}^{+}\right)\right\rangle /\left\langle v_{\alpha}^{2}\left(l_{3} \mathrm{Cl}^{-}\right)\right\rangle$ 


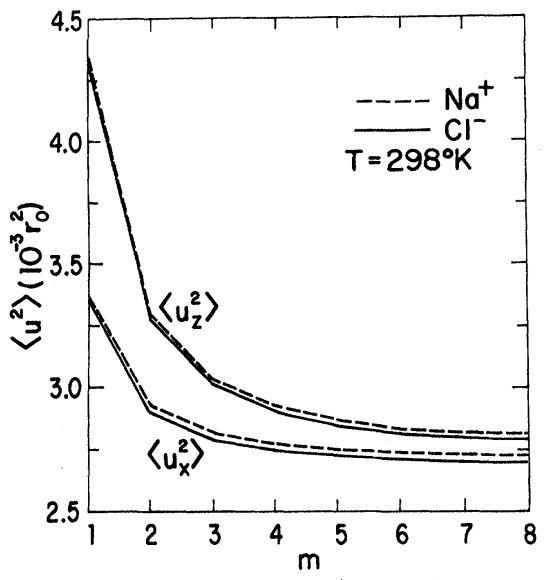

FIG. 1. Mean-square amplitudes of vibration $\left\langle u_{\alpha}^{2}\right\rangle$ for $\mathrm{Na}^{+}$and $\mathrm{Cl}^{-}$ions at $298^{\circ} \mathrm{K}$ as functions of distance from the surface. Here $m$ labels a plane of ions, with $m=1$ at the surface, and $r_{0}$ is the distance between nearest neighbors in the bulk (a $\mathrm{Na}^{+}$ion and a $\mathrm{Cl}^{-}$ion being nearest neighbors) and has the value of $2.814 \AA$.

$$
=\left(M_{\mathrm{Na}^{+}} / M_{\left.\mathrm{Cl}^{-}\right)^{-1}=1.54 \quad \text { as } T \rightarrow \infty,}\right.
$$

as borne out by Fig. 4.

It can be seen in Figs. 1-3 that the mean-square amplitudes for the $\mathrm{Na}^{+}$and $\mathrm{Cl}^{-}$ions are very nearly equal, particularly at high temperatures. In fact, one can show that $\left\langle u_{\alpha}^{2}\left(l_{3} \mathrm{Na}^{+}\right)\right\rangle$and $\left\langle u_{\alpha}^{2}\left(l_{3} \mathrm{Cl}^{-}\right)\right\rangle$ are exactly equal in the quasiharmonic approximation and the high-temperature limit for our model (i. e., a KRI model in which the $\mathrm{Na}^{+}$and $\mathrm{Cl}^{-}$ions in the same plane have the same static relaxation):

For this model, the force-constant matrix for the

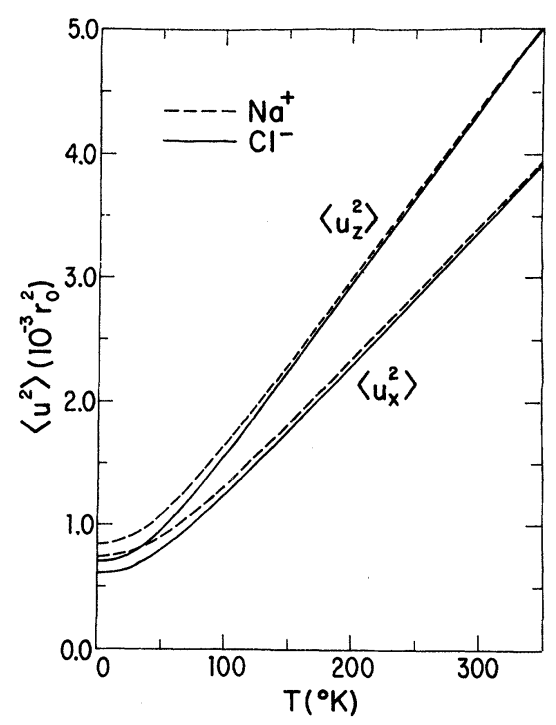

FIG. 2. Dependence of mean-square amplitudes at the surface (i.e., $m=1$ ) on the temperature $T$.

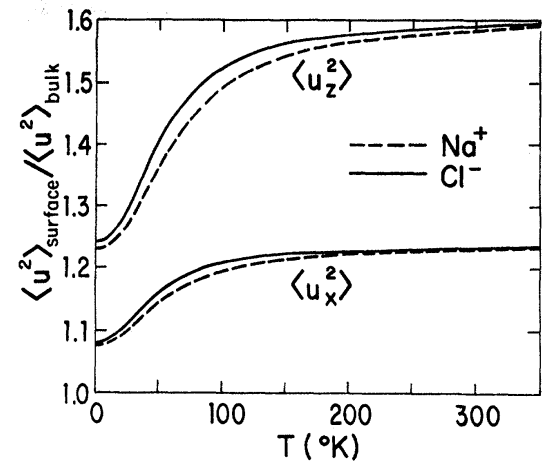

FIG. 3. Ratio of surface to bulk mean-square amplitudes as a function of temperature.

entire crystal ${ }^{16}$ is invariant under an interchange of the $\mathrm{Na}^{+}$and $\mathrm{Cl}^{-}$ions. It is known ${ }^{17}$ that, for an arbitrary harmonic system in the high-temperature limit, the mean-square amplitudes for a given particle are independent of the particle's mass and depend only on the force constants. ${ }^{18}$ Since, within the present model, the force constants are the same for $\mathrm{Na}^{+}$and $\mathrm{Cl}^{-}$ions in the same plane, the meansquare amplitudes must be equal in the high-temperature limit for $\mathrm{Na}^{+}$and $\mathrm{Cl}^{-}$ions in the same plane. ${ }^{19}$

Furthermore, an argument ${ }^{20}$ of Housley and Hess indicates that

$$
\begin{aligned}
\left\langle u_{\alpha}^{2}\left(l_{3} \mathrm{Na}^{+}\right)\right\rangle /\left\langle u_{\alpha}^{2}\left(l_{3} \mathrm{Cl}^{-}\right)\right\rangle & \approx\left(M_{\mathrm{Na}^{+}} / M_{\mathrm{Cl}^{-}}\right)^{-1 / 2} \\
& =1.24 \text { at } T=0^{\circ} \mathrm{K} .
\end{aligned}
$$

The actual calculated values for this ratio at the surface are 1.22 and 1.21 for vibrations in the $x$ and $z$ directions, respectively.

It can be seen that both of the above results, i.e.,

$$
\begin{array}{ll}
\left\langle u_{\alpha}^{2}\left(l_{3} \kappa^{\prime}\right)\right\rangle=\left\langle u_{\alpha}^{2}\left(l_{3} \kappa\right)\right\rangle & \text { for } T \rightarrow \infty, \\
\frac{\left\langle u_{\alpha}^{2}\left(l_{3} \kappa^{\prime}\right)\right\rangle}{\left\langle u_{\alpha}^{2}\left(l_{3} \kappa\right)\right\rangle} \approx\left(\frac{M_{\kappa^{\prime}}}{M_{\kappa}}\right)^{-1 / 2} \quad \text { for } T=0^{\circ} \mathrm{K},
\end{array}
$$

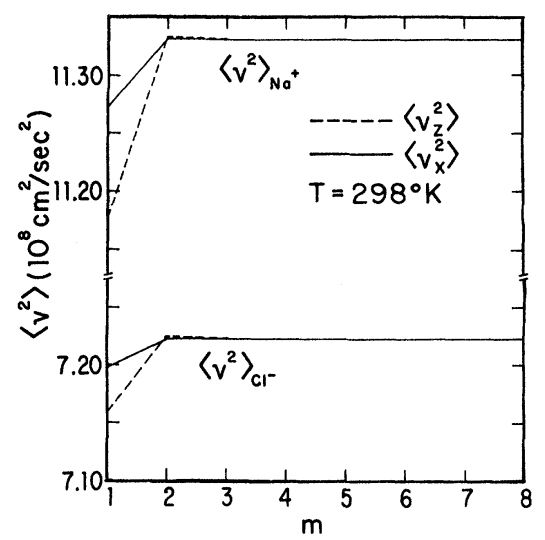

FIG. 4. Mean-square velocities $\left\langle v_{\alpha}^{2}\right\rangle$ for $\mathrm{Na}^{+}$and $\mathrm{Cl}^{-}$ions as functions of distance from the surface. 
TABLE I. Summary of experimental and theoretical values for bulk Debye-Waller parameters $B_{k}=8 \pi^{2}$ $\times\left\langle u_{x}^{2}(\kappa)\right\rangle$ of $\mathrm{NaCl}$ at room temperature (R. T.) and at low temperature (L. T.; $\leq 15^{\circ} \mathrm{K}$ ), where available.

\begin{tabular}{|c|c|c|c|c|}
\hline \multirow{2}{*}{$\begin{array}{l}B_{+} / \AA^{2} \\
\text { (R. T.) }\end{array}$} & \multirow{2}{*}{$\begin{array}{l}B_{-} / \AA^{2} \\
\text { (R.T.) }\end{array}$} & \multicolumn{2}{|c|}{$B_{+} / B_{-}$} & \multirow[b]{2}{*}{ Method } \\
\hline & & (R.T.) & (L.T.) & \\
\hline 1.81 & 1.49 & 1.21 & 1.22 & $\begin{array}{l}\text { Experimental } \\
\mathrm{x} \text { ray, } \text { powder }^{\mathrm{a}}\end{array}$ \\
\hline 1.89 & 1.61 & 1.17 & $\ldots$ & $x$ ray, single crystal ${ }^{b}$ \\
\hline 1.84 & 1.44 & 1.28 & $\cdots$ & $x$ ray, powder ${ }^{c}$ \\
\hline 1.64 & 1.33 & 1.23 & $\ldots$ & $x$ ray, single crystal $^{d}$ \\
\hline 1.63 & 1.42 & 1.15 & $\cdots$ & $\begin{array}{l}\text { Neutron diffraction, single } \\
\text { crystal }^{\circ}\end{array}$ \\
\hline 1.62 & 1.58 & 1.03 & 1.22 & $\begin{array}{l}\text { Theoretical } \\
\text { Deformation-dipole (DD), } \\
\text { R.T. parameters }\end{array}$ \\
\hline 1.59 & 1.47 & 1.08 & 1.26 & $\begin{array}{l}\text { DD next-nearest-neighbors, } \\
0^{\circ} \mathrm{K} \text { parameters }{ }^{1}\end{array}$ \\
\hline 1.56 & 1.35 & 1.15 & 1.32 & Shell model, quasiharmonic \\
\hline 1.71 & 1.70 & 1.00 & 1.22 & KRI model (present work) \\
\hline
\end{tabular}

${ }^{a}$ M. Linkoaho, Ann. Acad. Sci. Fennicae A284 (1968).

${ }^{b}$ S. Göttlicher, Acta Cryst. B24, 122 (1968).

'Reference 22.

${ }^{d}$ S. C. Abrahams and J. L. Bernstein, Acta Cryst. 18,926 (1965).

${ }^{\circ}$ H. A. Levy, P. A. Agron, and W. R. Busing, American Crystallography Association Meeting, Cambridge, Mass., Paper E-7, 1963 (unpublished).

${ }^{1}$ W. J. L. Buyers and T. Smith, J. Phys. Chem. Solids 29, 1051 (1968).

${ }^{8}$ Reference 21 .

are valid for any diatomic crystal provided that the force-constant matrix is invariant under an interchange of the two types of particles. This will be the case for models similar to that which we have used, i. e., models in which the ions are arranged in a $\mathrm{NaCl}$ structure and interact through pointcharge Coulomb forces and nearest-neighbor repulsive forces, and in which the positive and negative ions in a given plane undergo the same static relaxation.

In order to obtain some idea of the reliability of the present results, it is of interest to compare the present values for the bulk mean-square amplitudes $^{13}$ with experimental results and with theoretical results obtained from other, presumably more accurate, models. In Table I we give such a comparison in terms of the Debye-Waller parameters $B_{\kappa}=8 \pi^{2}\left\langle u_{\alpha}^{2}(\kappa)\right\rangle$ at room temperature and at low temperature. We refer to the paper by Reid and $\mathrm{Smith}^{21}$ for a fuller discussion of the comparison between experimental and theoretical determinations of the Debye-Waller factors for bulk ionic crystals. Here we mention only that the disparity between the two earlier experiments and the later experiments is probably due primarily to a lack of correction for thermal-diffuse scattering (TDS); Merisalo and Paakkari ${ }^{22}$ suggest that a correction for TDS of about $0.15 \AA^{2}$ should be added to the earlier results, which would bring them into much closer agreement with the later TDS-corrected work.

It would be interesting if LEED measurements on alkali-halide crystals could be carried out with the goal of studying the temperature dependence of the Bragg peaks. In the case of $\mathrm{NaCl}$, direct comparison with the results of Figs. 1-3 would be possible. In the case of other alkali halides, it would be possible to test (a) the qualitative conclusion that the ratio of surface to bulk mean-square amplitudes for vibrations normal to the surface should be relatively small, and (b) the degree to which the mean-square amplitudes for positive and negative ions in the same plane should be approximately equal at sufficiently high temperatures. The second point may provide an initial simplification for the approximate analysis of LEED data, in that it may be possible approximately to characterize each plane of ions by a single Debye-Waller factor.

Note added in proof. Although the KRI model is not quantitatively adequate for bulk mean-square amplitudes (MSA) - as shown by Table I-and hence cannot be expected to give accurate absolute predictions for surface MSA, such considerations leave open the question of how accurately the KRI model can predict surface MSA relative to those of the bulk. Preliminary results have now been obtained by the present authors for a shell model with surface relaxation neglected. For the present purpose, the differences between the KRI results and the preliminary shell-model results can best be summarized in terms of Fig. 3. There exist some noticeable but rather minor differences for the MSA components parallel to the surface. The principal differences appear in the curves for the MSA components normal to the surface: At $0^{\circ} \mathrm{K}$ the values of the shell-model curves for the $\mathrm{Na}^{+}$and $\mathrm{Cl}^{-}$ions are 1.23 and 1.32 , respectively; at $300^{\circ} \mathrm{K}$, they are, respectively, 1.54 and 1.73 .

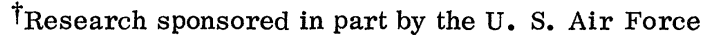
Office of Scientific Research (AFSC) under Grant No. 71-1973, and in part by the Robert A. Welch Foundation.

${ }^{1}$ For references, see R. E. Allen and F. W. de Wette, Phys. Rev. 188, 1320 (1969).

${ }^{2}$ S. Y. Tong and A. A. Maradudin, Phys. Rev. 181 , 1318 (1969).

${ }^{3}$ E. W. Kellermann, Phil Trans. Roy. Soc. London A238, 513 (1940).
}

${ }^{4}$ T. S. Chen, G. P. Alldredge, F. W. de Wette, and R. E. Allen, Phys. Rev. Letters 26, 1543 (1971).

${ }^{5}$ T. S. Chen, G. P. Alldredge, F. W. de Wette, and R. E. Allen, J. Chem. Phys. 55, 3121 (1971).

${ }^{6}$ T. S. Chen, R. E. Allen, G. P. Alldredge, and F. W. de Wette, Solid State Commun. $\underline{8}, 2105$ (1970). A number of unfortunate printing errors in this paper are corrected in an erratum: 9 , No. 16, xi (1971).

${ }^{7}$ F. W. de Wette and G. E. Schacher, Phys. Rev. 137, 
A78 (1965).

${ }^{8}$ A. A. Lucas, J. Chem. Phys. $\underline{48}, 3156$ (1968).

${ }^{9}$ Benson and co-workers have found that oversimplified models which allow different displacements for different ions tend to overestimate ionic displacements significantly. See G. C. Benson and K. S. Yun, in The SolidGas Interface, edited by E. A. Flood (Marcel Dekker, New York, 1967), Vol. I, Chap. 8, and references therein.

${ }^{10}$ E. R. Cowley, J. Phys. C $\underline{4}, 988$ (1971).

${ }^{11}$ R. E. Allen, F. W. de Wette, and A. Rahman, Phys. Rev. 179, 887 (1969).

${ }^{12}$ If the rate of thermal expansion is larger at the surface than in the bulk, there will be a larger decrease in the surface mean-square amplitudes.

${ }^{13} \mathrm{As}$ a check on these results, an independent calculation was performed for the bulk, and good agreement was obtained with the results for the center of the slab. (See Table I. )

${ }^{14}$ A. Ignatjevs, T. N. Rhodin, S. Y. Tong, B. I. Lundqvist, and J. B. Pendry, Solid State Commun. 9,
1851 (1971).

${ }^{15}$ Compare Fig. 2 of Ref. 4 with Fig. 10 of R. E. Allen, G. P. Alldredge, and F. W. de Wette, Phys. Rev. B $\underline{4}, 1661$ (1971).

${ }^{16}$ The force-constant matrix is the matrix $K$ of Ref. 17 .

${ }^{17}$ R. M. Housley and F. Hess, Phys. Rev。 146, 517 (1966).

${ }^{18}$ See Eqs. (10) and (13) of Ref. 17 [with a typographical error to be corrected in Eq. (13), so that $m_{k}^{-1} \rightarrow m_{k}$ ].

${ }^{19}$ The high-temperature equality of the Debye-Waller factors for different ions in bulk models having only nearest-neighbor interactions has been conjectured by E. R. Cowley and R. A. Cowley, Proc. Roy. Soc. (London) A292, 209 (1966).

${ }^{20}$ See the discussion on p. 521 (right-hand column) of Ref. 17.

${ }^{21}$ J. S. Reid and T. Smith, J. Phys. Chem. Solids 31 , $2689(1970)$, and references therein.

${ }^{22}$ M. Merisalo and T. Paakkari, Acta Cryst. $\underline{23}, 1107$ (1967).

\title{
Surface Modes of Vibration in the Rigid-Ion Model of $\mathrm{NaCl}^{\dagger}$
}

\author{
T. S. Chen, G. P. Alldredge, and F. W. de Wette \\ Department of Physics, University of Texas, Austin, Texas 78712 \\ and \\ R. E. Allen \\ Department of Physics, Texas A \& $M$ University, College Station, Texas 77843 \\ (Received 15 February 1972)
}

\begin{abstract}
A discussion is given of the complete spectrum of vibrational surface modes for the Kellermann rigid-ion model of $\mathrm{NaCl}$. The attenuation with distance from the surface is shown for representative surface and pseudosurface modes. We discuss one interesting feature which has not previously been pointed out: Symmetry requirements can lead to surface modes which are associated with only one species of ion $\left(\mathrm{Na}^{+}\right.$or $\left.\mathrm{Cl}^{-}\right)$at the surface.
\end{abstract}

\section{INTRODUCTION}

There have been several discussions ${ }^{1-5}$ of surface modes in the Kellermann rigid-ion (KRI) model of $\mathrm{NaCl}$, but as yet no complete and accurate discussion has been given. Although for many purposes the results for the KRI model will be superceded by calculations based on the shell model ${ }^{4}$ and other improved models which are currently in progress, we feel that it is appropriate to give a complete discussion of the surface-mode spectrum for the KRI model before passing on to the shell model. The results for the KRI model yield considerable insight into the qualitative features that can be expected generally of surface and pseudosurface modes in ionic crystals.

\section{RESULTS}

The method for calculating the vibrational modes is discussed in detail elsewhere. ${ }^{2}$ Our procedure differs from that of Ref. 2 in only two respects, as discussed previously., 6 The surface ions relax inward, rather than outward, by a small amount, and a more rapidly convergent method is used to evaluate the Coulomb lattice sums.

In Fig. 1, the calculated frequencies of vibration $\omega_{p}(\bar{q})$ are shown for a 15-layer slab. Since there are two particles per unit cell and three directions of vibration, there are $2 \times 3 \times 15=90$ frequencies for each two-dimensional wave vector $\bar{q}$; i.e., $p=1,2, \ldots, 90$, where $p$ distinguishes the different normal modes which are associated with a given $\bar{q}$. The values of $\bar{q}$ for which the frequencies were determined lie along the symmetry lines of the two-dimensional Brillouin zone, $\bar{\Gamma} \overline{\mathrm{X}}, \overline{\mathrm{X}} \overline{\mathrm{M}}$, and $\overline{\mathrm{M}} \bar{\Gamma}$, as shown in Fig. 2(c). In Figs. 2(a) and (b) we show the frequencies for values of $\bar{q}$ along two lines $(\bar{\Gamma} \bar{Y})$ which make angles of $30^{\circ}$ and $15^{\circ}$, respectively, with the line $\bar{\Gamma} \bar{M}$. (The endpoints of 